\title{
Zooming into primary breakup mechanisms of high-pressure automotive sprays
}

\author{
Valeri Kirsch*, Manuel A. Reddemann, Johannes Palmer, Reinhold Kneer \\ Institute of Heat and Mass Transfer, RWTH Aachen University, Augustinerbach 6, 52056 \\ Aachen, Germany \\ ${ }^{*}$ Corresponding author: kirsch@wsa.rwth-aachen.de
}

\begin{abstract}
In-cylinder mixture formation and combustion are highly influenced by primary breakup of injected fuel. Experimental investigation of this phenomenon directly at the outlet of a diesel injector requires a specialized transmitted light microscopy technique combined with a constant-pressure flow microscopy vessel. The method allows verification of the existence of an intact jet core for various states of injection and different fuels. The jet core is dominated by axisymmetric surface waves during the initial injection phase. By quantification of the wavelengths and comparison with existing breakup theories, boundary layer instabilities are identified as origin of surface waves. Boundary layer wavelengths are found to be larger for a higher fuel viscosity. An occasionally appearing non-cylindrical helical jet shape is visible during the injector's opening and closing phase. The helical jet shape is directly resulting from the nozzle outlet flow. Inner nozzle effects are found to be responsible for generation of the helical structure. A fuel dependence of the helical structure formation and its breakup could not be proved. Results also prove that fuel is exiting the nozzle even after the injector needle is closed, while air is simultaneously moving into the nozzle orifice.
\end{abstract}

\section{Keywords}

automotive spray, primary breakup, microscopy, boundary layer instability

\section{Introduction}

Underlying physics of primary jet breakup into poly-disperse sprays at engine-relevant conditions has been an important research topic for decades. Engine-relevant conditions require a complex experimental setup. Such a complex setup usually allows only limited optical accessibility of the region of interest. In addition, optical measurements of the liquid breakup of automotive sprays are difficult, because of the phenomenon itself: Injection pressures up to $300 \mathrm{MPa}$ generate billions of liquid structures (jet, ligaments, droplets) with high velocities (up to $800 \mathrm{~m} / \mathrm{s}$ ) and microscopic scales (up to $1 \mu \mathrm{m}$ ). Multiply scattered light at the gas-liquid interface of these structures increase the optical density of the spray by such a degree that almost no information is gained in the near-field of the nozzle based on classical visualization techniques [1]. As a consequence, scaled generic nozzles are used for fundamental primary breakup investigations. Though, based on generic nozzles, engine-relevant dimensionless numbers and nozzle effects (such as cavitation) cannot be comprehensively reproduced. Therefore, automotive nozzles must be investigated for a complete primary breakup description.

To enable optical investigation of primary breakup even for engine-relevant injection conditions, several experimental methods are specifically developed, e.g. Ballistic Imaging [2] or X-Ray Absorption [3]. Besides these advanced methods, conventional microscopy with optimized optics and adjusted illumination sources have shown great capability for detailed investigations of primary breakup directly at the nozzle orifice. Reddemann et al. [4, 5] investigated primary breakup of diesel jets under atmospheric conditions with a double-frame transmitted light microscope (DF-TLM). Further publications also show microscopic investigations of the primary breakup for increased ambient conditions $[6,7,8]$.

In this work, a DF-TLM system is used for microscopic imaging of the primary breakup at cold engine-like ambient densities. Images are recorded directly at the nozzle outlet of a diesel injector. Focus of the analysis lies on the transient moments of the injection (the opening and closing phase). Three different fuels (diesel, 1-octanol, di-n-butyl ether) are utilized, to investigate the effect of a wide range of dimensionless numbers (Ohnesorge, Reynolds) on the jet breakup. Recorded images deliver highly resolved information of the breakup structures, which are evaluated in size, shape and velocity. These values are used for comparison with physical theories that could explain the observed breakup mechanisms.

\section{Material and methods}

An upgraded version of the DF-TLM system and the constant-pressure flow microscopy (CPF-M) vessel presented by Reddemann et al. [4, 7] are used for primary breakup investigations directly at the nozzle orifice (figure 1). The optical system of TLM is adjusted for automotive sprays, covering a numerical aperture of NA=0.16 and a resolution of $2 \mu \mathrm{m}$ (Abbe criterion [9]). This configuration leads to a depth of field of DOF=70 $\mu \mathrm{m}$ (airy disk diameter as criterion for circle of confusion). The CPF-M vessel enables investigations at ambient pressures up to $5 \mathrm{MPa}$ at atmospheric temperature $(\mathrm{T}=293 \mathrm{~K})$. The illumination source, a pulsed diode laser (Cavilux Smart), produces almost incoherent light pulses ${ }^{1}$ with a duration of $10 \mathrm{~ns}$ and a wavelength of $640 \mathrm{~nm}$. The light source suppresses speckles,

${ }^{1}$ The light source has a coherence length of about $80 \mu \mathrm{m}$. Thus, this light source can be treated as incoherent for most observed structures in this work. 


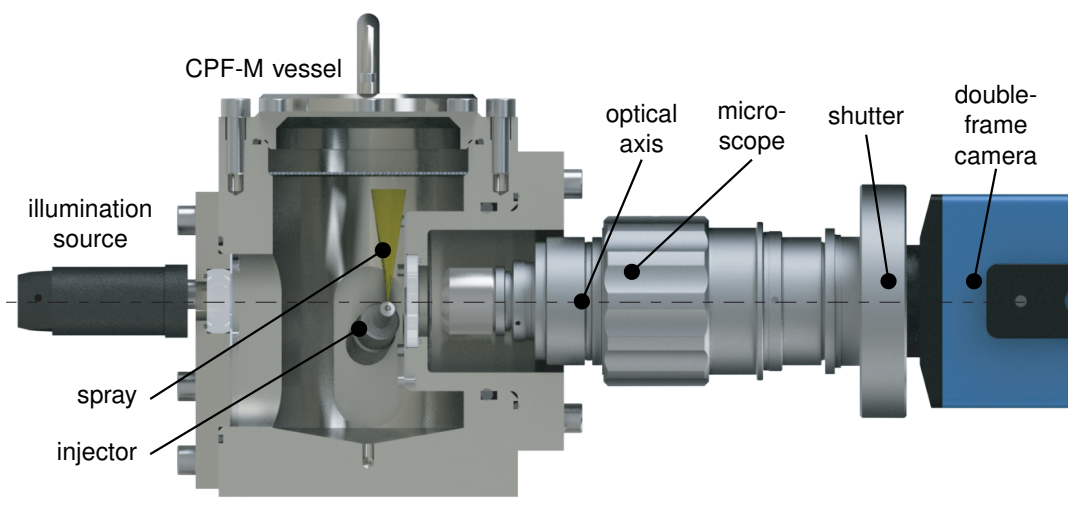

Figure 1. Combined setup of the Double Frame Transmitted Light Microscope (DF-TLM) and the Constant-Pressure Flow Microscopy (CPF-M) chamber.

chromatic aberrations and motion blur up to a spray velocity of $100 \mathrm{~m} / \mathrm{s}^{2}$. A double-frame CCD-Camera (LaVision Flowmaster) delivers not only shapes and sizes at the nozzle near-field of the primary breakup structures, but also offers possibility to measure displacements of the structures during an inter-framing time of $1 \mu \mathrm{s}$. Double-frame images allow velocity determination under consideration of the magnification factor of the optical system $(M=6.7)$. Central element of the experimental setup is a piezo-injector with a 3-hole nozzle $(D=140 \mu \mathrm{m}$, length-to-diameter ratio $=7.32$, inlet to outlet ratio $=1.1$, midi sac-hole). Alignment of injector and optical axis is shown in figure 1 . An injection pressure of $100 \mathrm{MPa}$ and an energizing duration of $1800 \mu \mathrm{s}$ are chosen for the investigation. Compared to real engine applications, this long energizing duration ensures a steady-state injection phase. 1-octanol, di-n-buthyl ether (DnBE) as alternative fuels and diesel are used to cover a wide range of Reynolds and Ohnesorge numbers. Physical properties, dimensionless numbers and operating conditions (OC) are shown in table 1.

Table 1. Fuel properties at $293 \mathrm{~K}$ and dimensionless numbers at investigated operating conditions (OC).

\begin{tabular}{|c|c|c|c|c|c|c|c|c|}
\hline & OC & $\begin{array}{c}\rho \\
\mathrm{kg} / \mathrm{m}^{3}\end{array}$ & $\begin{array}{c}\eta \\
\mathrm{mPas}\end{array}$ & $\begin{array}{c}\sigma \\
\mathrm{mN} / \mathrm{m}\end{array}$ & $\begin{array}{c}p_{v} \\
\mathrm{kPa}\end{array}$ & $\begin{array}{c}\rho^{*} \\
-\end{array}$ & $\begin{array}{c}R e \\
-\end{array}$ & $\begin{array}{c}O h \\
-\end{array}$ \\
\hline $\begin{array}{l}\text { diesel } \\
\text { EN590 }\end{array}$ & $\begin{array}{l}d i 1 \\
d i 2\end{array}$ & 833 & 3 & 20.5 & $<1$ & $\begin{array}{c}2.9 \mathrm{e}-3 \\
11.5 \mathrm{e}-3\end{array}$ & $\approx 19000$ & 0.061 \\
\hline 1-octanol & $\begin{array}{l}o c 1 \\
o c 2\end{array}$ & 826 & 8.6 & 26.6 & 0.01 & $\begin{array}{c}2.9 \mathrm{e}-3 \\
11.6 \mathrm{e}-3\end{array}$ & $\approx 6600$ & 0.155 \\
\hline DnBE & $\begin{array}{l}d n 1 \\
d n 2\end{array}$ & 768 & 0.69 & 22.6 & 0.82 & $\begin{array}{c}3.1 \mathrm{e}-3 \\
12.5 \mathrm{e}-3\end{array}$ & $\approx 79500$ & 0.014 \\
\hline
\end{tabular}

\section{Results and discussion}

An exemplary time series of an 1-octanol injection is shown in figure 2. Regardless of the injection phase, a connected jet core is visible directly at the nozzle orifice. The injection process can be subdivided into five characteristic phases: I) start of injection (needle opening) shortly after start of energizing ( $t=295 \mu \mathrm{s})$, II) acceleration during needle lifting $(\mathrm{t}=500 \mu \mathrm{s})$, III) steady-state $(\mathrm{t}=2500 \mu \mathrm{s})$, IV) deceleration during needle subsiding $(\mathrm{t}=4500 \mu \mathrm{s})$ and $\mathrm{V})$ end of injection after the needle is closed ${ }^{3}(\mathrm{t}=4750 \mu \mathrm{s})$. Injector opening and closing moments vary slightly, depending on the utilized fuel. The needle response depends strongly on viscosity of injected fuel and can be determined by injection rate measurements. For the low viscosity fuel $\mathrm{DnBE}$, needle closing occurs $\mathrm{t}=4250 \mu$ s and for high viscosity

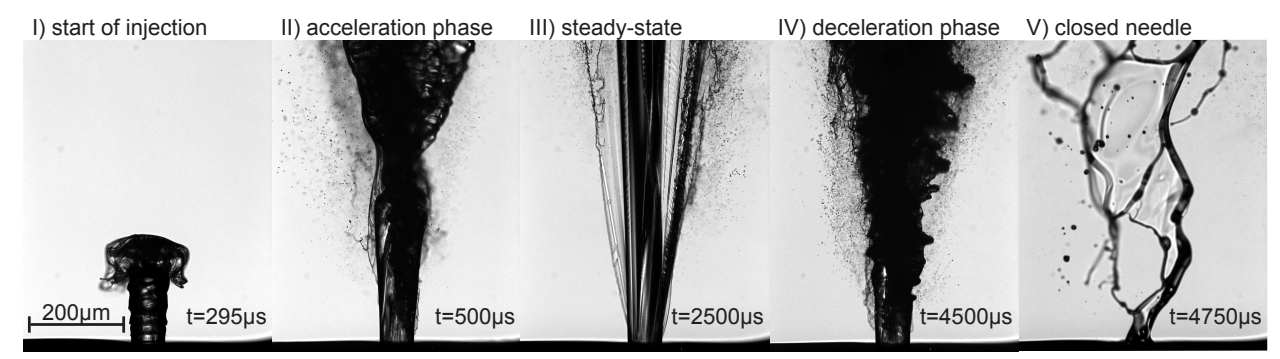

Figure 2. Exemplary images of the primary breakup process at different injection phases. Injected fuel: 1-octanol, injection pressure: $100 \mathrm{MPa}$, ambient density: $2.4 \mathrm{~kg} / \mathrm{m}^{3}$ and ambient temperature: $293 \mathrm{~K}$.

\footnotetext{
${ }^{2}$ Based on the criterion of one pixel displacement on the image plane.

${ }^{3}$ Additional injection rate measurements performed with a Bosch-type method rate meter [10] for the same injection and ambient conditions, proof needle closing at a time of $t \approx 4600 \mu$ s after start of energizing
} 
fuel 1 -octanol needle closing is reached later at $t=4600 \mu \mathrm{s}$. In the following, breakup mechanisms of the injection phases are described and analyzed in detail, exemplary for 1-octanol.

\section{I) Start of injection:}

Figure 3 shows images of the start of injection for three fuels (1-octanol, diesel and DnBE) for two different ambient densities $\left(\rho=2.4\right.$ and $\left.9.6 \mathrm{~kg} / \mathrm{m}^{3}\right)$. Even if time after energizing is kept constant, the jet penetration is not identical but varies from 300 to $450 \mu \mathrm{m}$ ). The typical jet tip shape during start of injection is a spheroidal cap and is basically shown for all images of figure 3. However, in case of DnBE at low ambient density, the spheroidal cap is covered with a pre-ligament. Just below the spheroidal tip axisymmetric surface waves are also visible in all shown images. Wavelengths of those disturbances are manually measured and illustrated in figure 3 . Measured wavelengths are fuel-dependent and decrease in length from 1-octanol to diesel to DnBE. Occurring wavelengths of each fuel are independent of ambient density. Tip velocities are determined with double-frame images and are illustrated also in figure 3. Calculated velocities for the higher ambient density $(\mathrm{v} \approx 35-61 \mathrm{~m} / \mathrm{s})$ are lower compared to the velocities at the lower ambient density $(\mathrm{v} \approx 64-68 \mathrm{~m} / \mathrm{s})$. It is important to note for later wavelength evaluation that for an ambient density of $\rho=2.4 \mathrm{~kg} / \mathrm{m}^{3}$ tip velocities are almost the same. However, images presented in figure 3 are selected, because they give insight into the process of a more or less laminar jet propagation during start of injection. The same injection conditions may also produce a turbulent jet tip with many small droplet and ligaments, but neither a spheroidal cap nor axisymmetric surface waves. Turbulent jet tips require further information for interpretation and are discussed in the section of the closed needle phase.
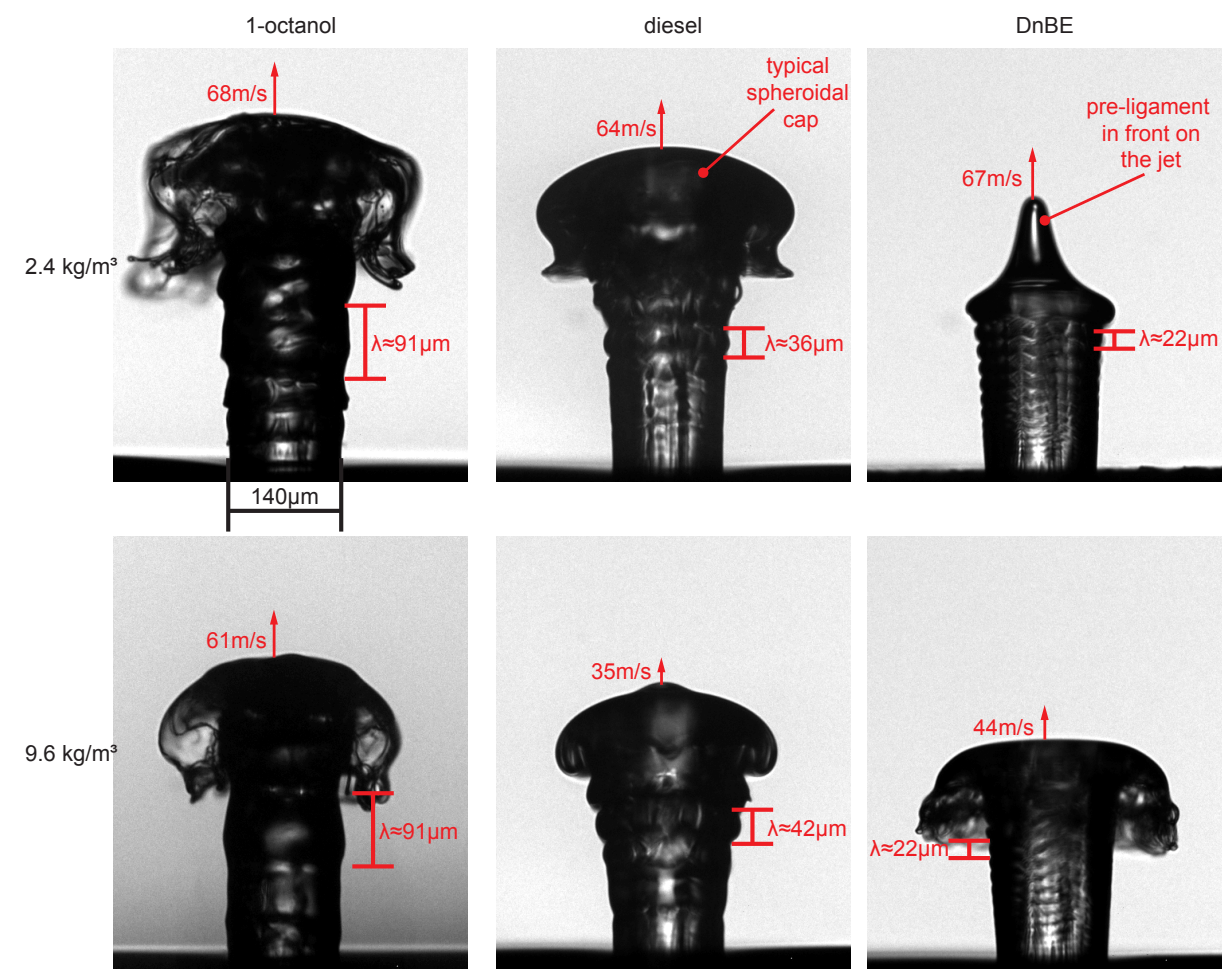

Figure 3. Images of the start of jet penetration of the three fuels 1-octanol, diesel and DnBE for two ambient densities $2.4 \mathrm{~kg} / \mathrm{m}^{3}$ and $9.6 \mathrm{~kg} / \mathrm{m}^{3}$. Axisymmetric waves are present at almost the same axial nozzle distance, but with different wavelengths. Time after energizing: 285 - $295 \mu \mathrm{s}$, injection pressure: $100 \mathrm{MPa}$, ambient temperature: $293 \mathrm{~K}$.

The spheroidal cap at the jet tip, with and without pre-ligaments, are also observed and described for laminar jet tips by other authors, e.g. Crua et al. [11], Badock and Wirth [12], Busch [13], Liu et al. [14], Hult et al. [15] or Schugger [16]. Crua et al. [11] explains the existence of a spheroidal cap at the jet tip by an interaction of adhesion forces at the nozzle surface and rapidly increasing axial jet velocity: fresh fuel from inside the nozzle hole pushes the jet forwards into the atmosphere until inertia of the jet tip compensates the adhesion force and the spheroidal cap detaches from the nozzle surface. Pre-ligaments in front of the spheroidal cap are explained by a slipstream effect, which propels the central pre-ligament ahead of the jet into the atmosphere [11].

Occurrence of axisymmetric surface waves during jet formation have been rarely discussed for automotive sprays so far. In a more general context of low Reynolds and Weber number, formation of axisymmetric surface disturbances are explained by two possible reasons: i) Kelvin-Helmholtz instabilities or ii) boundary layer instabilities [17]. It is commonly known that wavelengths that occur due to Kelvin-Helmholtz instabilities behave inversely proportional to changes in ambient density $[18,17]$. Results presented in figure 3 are in contradiction to this theory. Therefore, axisymmetric surface waves cannot be caused by Kelvin-Helmholtz instabilities. Instead, bounrary layer instabilities could be an explanation for the origin of the surface waves. Boundary-layer instabilities are developed, due to jet velocity profile relaxation [17]. According to Brennen's analytic boundary layer theory, the ratio between mean jet velocity $u$ and instability frequency $f$ can be expressed as function of boundary layer momentum thickness $\delta_{2}$ and 
dimensionless frequency $\gamma$, which in turn can be approximated as boundary layer instability wavelength $\lambda_{B L}$ [19]:

$$
\frac{u}{f}=\frac{2 \cdot \pi \cdot \delta_{2}}{\gamma} \approx \lambda_{\mathrm{BL}}
$$

For a laminar case, dimensionless frequency $\gamma$ is given with a maximum growth rate of 0.175 . Under the assumption of a Blasius's solution for laminar flow fields [17], boundary layer momentum thickness depends on fuel properties (density and viscosity) and jet velocity (injection pressure), only. These influences are usually taken into account on the basis of the steady-state Reynolds number. Such an assumption is not allowed in the current case due to the transient behavior of the jet (increasing velocity, uncompleted velocity profile relaxation). Nevertheless, analyzing the illustrated images in figure 3 at the ambient density of $\rho=2.4 \mathrm{~kg} / \mathrm{m}^{3}$ show an almost constant jet tip velocity. Thus, boundary layer momentum thickness and boundary layer instability wavelengths depend on fuel properties, only. Fuel viscosity must have a major influence on the wavelengths, because viscosity differences between the three fuels are much larger compared to differences in density (compare table 1). If boundary layer instabilities and wavelengths are only depending on the fuel viscosity, a smaller boundary layer momentum thickness is expected and a small boundary layer wavelength for a lower viscosity should be visible in the recorded images (compare equation 1). This is clearly the case (see figure 3). As a result, observed surface waves are in in good agreement with boundary layer instability theory.

\section{II and IV) Acceleration and deceleration phase:}

Figure 4 shows the acceleration phase during needle lifting. A rough jet surface with many surface disturbances and structures (sheets, ligaments and droplets) around the jet core can be observed in both double-frame images (top). Directly at the nozzle outlet, the jet core has glare lines on its surface (see magnification). Glare lines run on the jet's surface in an axial-tangential direction. Further downstream, optical density is increased due to surface disturbances and structures around the jet in such a degree that almost no details about the inner jet core are visible. The outer shape of the jet shows frequent constrictions and expansion along the flow direction. Same observations are found for the deceleration phase of the jet during needle subsiding (not shown in the figure). In the following, both phases are analyzed simultaneously, due to their physical similarity.

Cavitation is assumed to be responsible for the high amount of surface disturbances during this injection phases. Turbulence is generated by cavitation, which in turn disturbs the jet surface [20]. Near nozzle glare lines observed on the jet surface indicate a tangential velocity share in the jet flow field. Proximity of the glare lines to the nozzle hole suggests that the tangential flow is caused by a vortex flow inside the nozzle hole. This vortex flow can be caused by string cavitation inside the sac hole [21, 22] or by an off-centered needle position [23]. Soteriou et al. [24] showed that centrifugal forces caused by such a vortex flow is able to shape the jet core into a helical shape. Such a three-dimensional helical shape cannot be fully proven just by analyzing the in this work taken two dimensional double-frame images. Nevertheless, the hypothesis of such a helical structure is reasonable for the observed jet properties. A schematically illustration of a here maybe observed left-handed helical jet shape is shown at the right bottom of figure 4. Main constriction and expansion of a hypothetical helix along the flow direction can be approximated as shown in the top left image of figure 4. The mode of such a helix cannot be determined with the given limited informations. However, as shown by Kubitschek and Weidman [25] a helical shaped jet is accompanied by formation of liquid sheet structures. Sheet structures start to form at the outer curvature of the hypothetical helix due to centrifugal forces. The same centrifugal forces cause a subsequent thinning of the sheet structures. The curvature of the helix is thus decreased for a larger distance from nozzle outlet. Thinning of the sheet structures finally ends in breakup and generation of ligaments and droplets with a flight path in radial-axial direction. Similar effects can be observed at both double-frame images in figure 4. The only difference is that breakup of in this work observed sheet structures is most likely not only caused by centrifugal and surface tension forces but also by aerodynamic forces. These aerodynamic forces disturb the sheet surface until the sheet structure cannot be hold together by cohesion forces anymore and break up in ligaments and droplets.

\section{III) Steady-state of the injection:}

The steady-state phase of the injection has already been analyzed in our previous publication [26] and is therefore not topic of this work. For the same fuels, injection and ambient conditions, a rippled structure and transversal disturbances on the jet surface are found. Rippled structures occurring directly at the nozzle hole are independent of ambient conditions and fuel properties. The jet cross-section is shaped by this rippled structures into a star-like polygon. An explanation for transversal disturbances on the jet surface have not been found so far, but will be investigated in a future work.

\section{V) Closed needle:}

Figure 5 shows double-frame images of the injection shortly after needle closing. At this injection state, fuel in the vessel is connected to the nozzle hole. Mostly large connected liquid structures are visible across the entire image. These connected structures consist of large ligaments (diameters up to $100 \mu \mathrm{m}$ ), which are often connected by sheets (sheet thickness $\leq 3 \mu \mathrm{m})^{4}$. On most of these sheet structures possible interference patterns are visible. Scattered big droplets $(d \approx 5-35 \mu \mathrm{m})$ around the structures and some bubbles $\left(d_{b} \approx 35 \mu \mathrm{m}\right)$ inside the structures ${ }^{5}$

\footnotetext{
${ }^{4}$ The sheet thickness can be approximated by measuring the size of the smallest ligament connected to the sheet with a diameter of approximately $3 \mu \mathrm{m}$. The ligament has to be larger in diameter as the sheet thickness due to surface tension forces.

${ }^{5}$ Three reasons justify the conclusion that the structures inside the large ligaments have to be bubbles: 1) If those round structures are considered to be droplets, some of the droplets have to be formed inside the nozzle hole. Since some of them are really close to the nozzle hole. It is very
} 


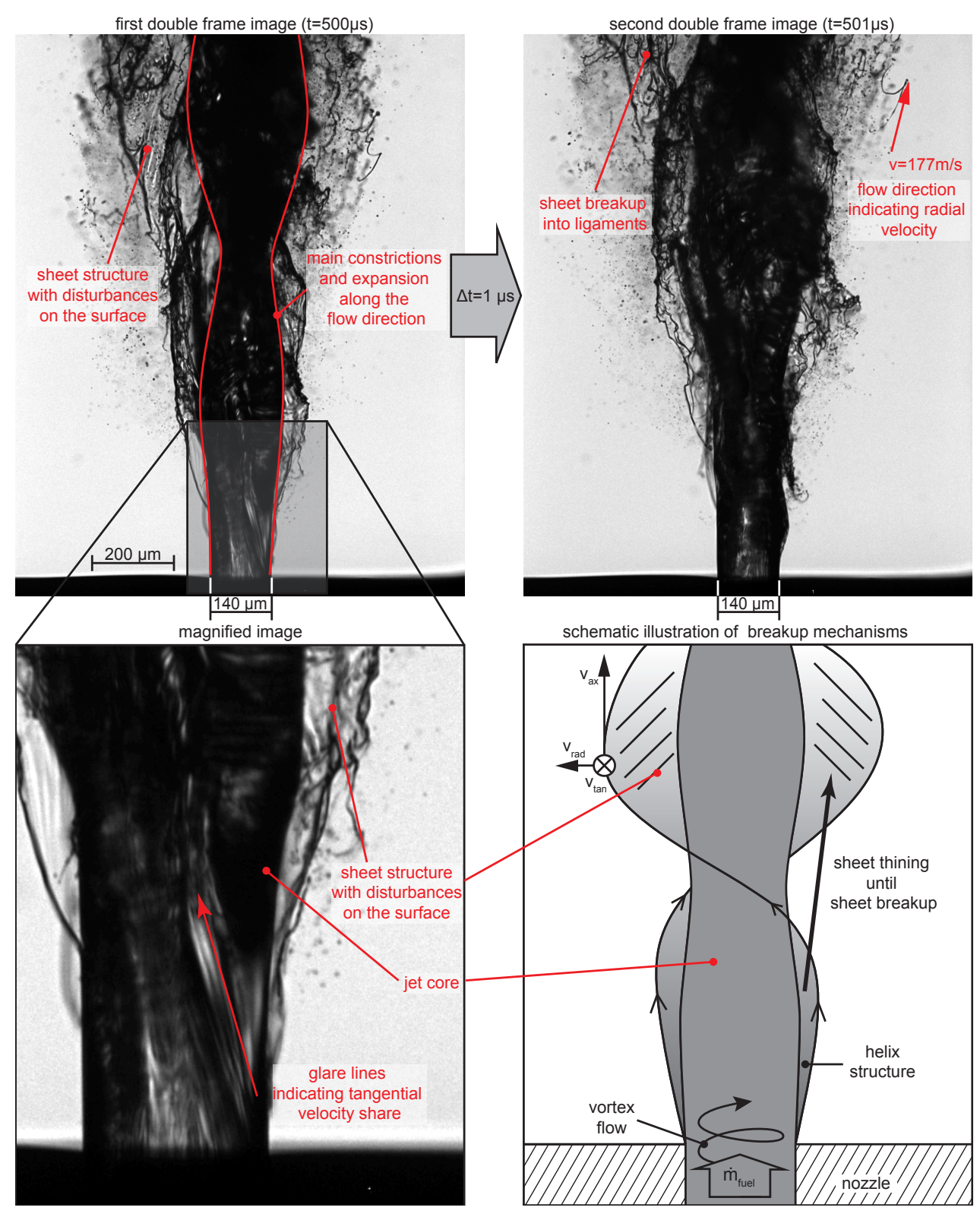

Figure 4. Double-frame images of the phase during the needle opening with a schematic illustration of observed mechanisms. Time after energizing: $500 \mu \mathrm{s}$, Injected fuel: 1 -octanol, injection pressure: $100 \mathrm{MPa}$, ambient density: $2.4 \mathrm{~kg} / \mathrm{m}^{3}$ and ambient temperature: $293 \mathrm{~K}$.

are present as well. A high amount of small droplets $(d \approx 1-4 \mu \mathrm{m})$ can be observed in some finite areas (magnification in figure 5). By analyzing double-frame images, a tangential velocity share and maximum velocities up to $v \leqq 22 \mathrm{~m} / \mathrm{s}$ can be calculated. With further nozzle distance, velocities are increasing. Almost no fluid velocity is measurable directly at the nozzle orifice. The orifice is not fully covered with fuel, but the surface around the orifice is partly covered with liquid.

The uninterrupted flow of fuel after needle closing may be explained by the remaining injection inertia. The inertia propels fuel out of the nozzle hole until friction forces fully dissipate the inertia force inside the nozzle hole (balance of forces in figure 5, bottom right). Once this state is reached, structures close to the nozzle hole are thinned due to surface tension forces. Eventually, the liquid structure will tear apart at this thinned position. Structures downstream the tear-off edge separate from remaining fuel and move further into the vessel. Fuel upstream the tear-off edge remains attached to the nozzle and stays there until the next injection starts. Fuel attaches to the nozzle due to adhesion forces.

The remaining radial and tangential inertia of liquid structures moving inside the vessel tears the structure apart into many separate secondary ligaments. Connecting sheets in between the structures are thinning until they fragment into small ligaments and droplets due to the increasing distance between the sheet surrounding ligament

unlikely that droplets will form inside the nozzle and fly into ambiance without sticking to other structures. 2) The observed round structures follow the ligaments the entire observed time. This is only possible if they are included into the ligaments. 3) Some of the big round structures are constantly deformed to a longitudinal shape. The only possible reason for such a deformation even at the observed small velocities is surface tension forces caused by an enclosing surface of the bigger sized ligaments. 


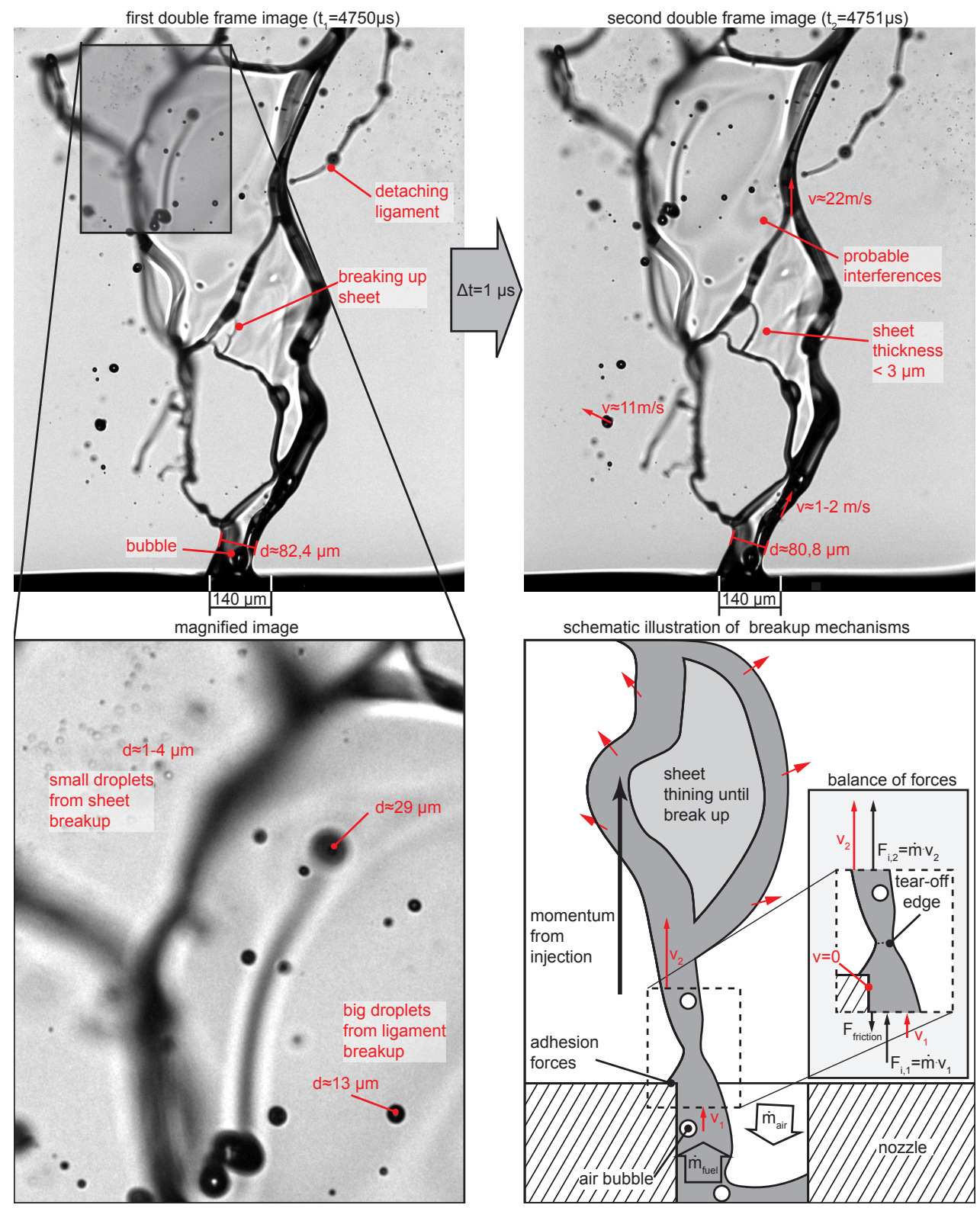

Figure 5. Double-frame images of the phase shortly after the needle closed with a schematic illustration of observed mechanisms. Time after energizing: $4750 \mu \mathrm{s}$, Injected fuel: 1 -octanol, injection pressure: $100 \mathrm{MPa}$, ambient density: $2.4 \mathrm{~kg} / \mathrm{m}^{3}$ and ambient temperature: $293 \mathrm{~K}$.

structures. Size of those developing small ligaments and droplets has to be in the same order of the sheet thickness. This means that a high amount of small droplets and ligaments $(d<4 \mu \mathrm{m})$ develop in a finite area by sheet breakup. Big droplets $(d>4 \mu \mathrm{m})$ in turn, develop by fragmentation of large ligaments. Most of the observed fuel mass at this phase remains as big droplets or big sized ligaments inside the vessel.

A not fully covered nozzle hole can be explained as follows: As soon as the injector needle is closed, fuel from inside the injector cannot flow into the nozzle. As a result, air moves inside the nozzle hole as compensation for fuel that has left the nozzle after needle closing. Once inertia is too small to propel further fuel into the vessel, air inside the nozzle is trapped until start of a consecutive injection. Similar observations where are observed by Blessing [20] and Ghiji et al. [27].

Observed bubbles inside liquid structures must consist either of i) fuel vapor or ii) ambient gas. Fuel vapor inside the nozzle hole usually develops at automotive injections by cavitation. Cavitation is caused by high fuel velocities with a hydrostatic pressure below the vapor pressure of the injected fuel [28]. For a closed needle, the total pressure inside the nozzle hole can be approximated with the ambient pressure of $0.2 \mathrm{MPa}$. Considering this fact, velocity inside the nozzle hole has to be at least $22 \mathrm{~m} / \mathrm{s}$ for cavitation to set in (approximated with Bernoulli equation). As shown in figure 5, fuel velocity decreases with lower nozzle distance even below $2 \mathrm{~m} / \mathrm{s}$ until no movement is measurable anymore directly at the nozzle hole. Over time, inertia inside the nozzle hole is dissipated by friction forces. As a result, velocity inside the nozzle hole has to be smaller than the velocity outside the nozzle hole. With this low fluid velocity observed, bubbles inside liquid structures cannot be caused by cavitation. As a result, bubbles must consist 
of air that has just moved into the nozzle.

Air that moved into the nozzle, may exit the nozzle again immediately shaped as air bubbles. Air bubbles trapped in liquid structures are streaming out the nozzle as long as inertia of the injection is not completely dissipated. Remaining air inside the nozzle is most likely responsible for the varying initial jet shape of a subsequent injection. Air bubbles trapped inside the liquid fuel are probably compressed during the fuel acceleration due to inertia forces. As soon as air bubbles leave the nozzle hole, pressure drops significantly and bubbles abruptly decompress and disturb the jet. This mechanism was analysed in detail by Ghiji et al. [27]. However, this latter mechanism has not been shown in this work.

\section{Summary}

High resolution images of the near nozzle primary breakup region are shown for increased ambient densities. Two alternative fuels (1-octanol, DnBE) and diesel are investigated. Images are recorded by a double-frame transmitted light microscope, optimized just for the investigation of the primary breakup region of automotive jets. Five typical injection phases are resolved and described in detail: start of injection (needle opening), acceleration phase during during needle rising, steady-state, deceleration phase during needle subsiding and end of injection after the needle is closed. A spheroidal jet tip and axisymmetric surface waves are often visible during the initial injection phase. The analysis of the surface wavelengths suggests that those waves are caused by boundary layer instabilities. During needle opening and closing, a vortex flow caused by string cavitation or an off-centered needle position shapes the jet into a helical structure. The steady-state of the injection is described in a previous work and is therefore not a topic in this work. After the injector needle is closed, fuel still leaves the nozzle hole due to the not fully dissipated momentum of the injection. As compensation for fuel that still exits the nozzle hole, air is streaming into the nozzle hole. This mechanism is most likely responsible for the varying jet shape during the initial state of a consecutive injection. Fuel influences are found with high certainty for the axysmmetric jet waves only. For other phenomena, such as the helical jet shape ligament and sheet-breakup after needle closing, reproducibility is low and no fuel influences are identified.

\section{Acknowledgement}

This work was performed as part of the Cluster of Excellence "Tailor-Made Fuels from Biomass", which was funded by the Excellence Initiative of the German federal and state governments to promote science and research at German universities.

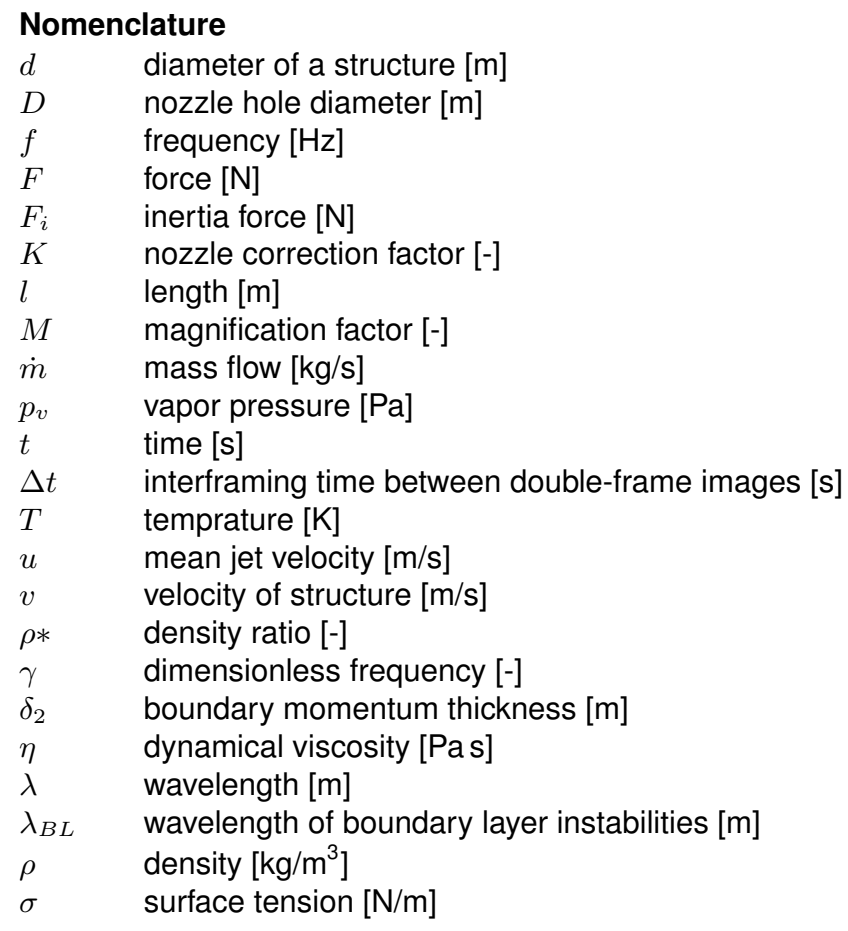

\section{References}

[1] Linne, M., 2013. "Imaging in the optically dense regions of a spray: A review of developing techniques". Progress in Energy and Combustion Science, 39(5), pp. 403-440.

[2] Linne, M., Paciaroni, M., Hall, T., and Parker, T., 2006. "Ballistic imaging of the near field in a diesel spray". Experiments in Fluids, 40(6), pp. 836-846. 
[3] Yue, Y., Powell, C. F., Poola, R., Wang, J., Lai, M.-C., and Parrish, S. E., 2001. "Quantitative Measurements of Direct-Injection Gasoline Fuel Sprays in Near-Nozzle Region Using Synchrotron X-Ray". In SAE 2001 World Congress, SAE Technical Paper Series.

[4] Reddemann, M. A., Mathieu, F., and Kneer, R., 2013. "Transmitted light microscopy for visualizing the turbulent primary breakup of a microscale liquid jet". Experiments in Fluids, 54(11).

[5] Reddemann, M. A., Mathieu, F., and Kneer, R., 2013. "Zooming into the primary breakup region of enginerelated sprays". In ILASS - Europe 2013, 25th European Conference on Liquid Atomization and Spray Systems, Chania, Greece, 1-4 September 2013.

[6] Crua, C., Shoba, T., Heikal, M., Gold, M., and Higham, C., 2010. "High-Speed Microscopic Imaging of the Initial Stage of Diesel Spray Formation and Primary Breakup". In SAE 2010 World Congress \& Exhibition, SAE Technical Paper Series.

[7] Reddemann, M. A., Kirsch, V., and Kneer, R., 2014. "Transmitted light microscopy of primary breakup for engine-relevant ambient densities". In ILASS - Europe 2014, 26th Annual Conference on Liquid Atomization and Spray Systems, 8-10 Sep. 2014, Bremen, Germany.

[8] Shoba, T., Crua, C., Heikal, M. R., and Gold, M., 2011. "Optical Characterisation of Diesel, RME and Kerosene Sprays by Microscopic Imaging". In ILASS - Europe 2011, 24th European Conference on Liquid Atomization and Spray Systems, Estoril, Portugal, September 2011.

[9] Abbe, E., 1873. "Beiträge zur Theorie des Mikroskops und der mikroskopischen Wahrnehmung". Archiv für mikroskopische Anatomie, 9(1), pp. 413-418.

[10] W. Bosch, 1964. "Der Einspritzgesetz-Indikator, ein neues Meßgerät zur direkten Bestimmung des Einspritzgesetzes von Einzeleinspritzungen". MTZ - Motortechnische Zeitschrift, 25(7), pp. 268-282.

[11] Crua, C., Heikal, M. R., and Gold, M. R., 2015. "Microscopic imaging of the initial stage of diesel spray formation". Fuel, 157, pp. 140-150.

[12] Badock, C., Wirth, R., Fath, A., and Leipertz, A., 1999. "Investigation of cavitation in real size diesel injection nozzles". International Journal of Heat and Fluid Flow, 20(5), pp. 538-544.

[13] Busch, R., 2001. "Untersuchung von Kavitationsphänomenen in Dieseleinspritzdüsen". PhD thesis, Hannover, Hannover.

[14] Liu, Z., Im, K.-S., Wang, Y., Fezzaa, K., Xie, X.-B., Lai, M.-C., and Wang, J., 2010. "Near-Nozzle Structure of Diesel Sprays Affected by Internal Geometry of Injector Nozzle: Visualized by Single-Shot X-ray Imaging". In SAE 2010 World Congress \& Exhibition, SAE Technical Paper Series.

[15] Hult, J., Simmank, P., Matlok, S., Mayer, S., Falgout, Z., and Linne, M., 2016. "Interior flow and near-nozzle spray development in a marine-engine diesel fuel injector". Experiments in Fluids, 57(4), p. 195.

[16] C. Schugger, 2007. "Experimentelle Untersuchung des primären Strahlzerfalls bei der motorischen Hochdruckeinspritzung". PhD thesis, Rheinisch Westfälische Technische Hochschule Aachen, Aachen.

[17] Yoon, S. S., and Heister, S. D., 2003. "Categorizing linear theories for atomizing round jets". Atomization and Sprays, 13(5-6), pp. 499-516.

[18] Reitz, R. D., and Bracco, F. V., 1982. "Mechanism of atomization of a liquid jet". Physics of Fluids, 25(10), p. 1730.

[19] Brennen, C., 1970. "Cavity surface wave patterns and general appearance". Journal of Fluid Mechanics, 44(01), p. 33.

[20] Blessing, M., 2004. "Untersuchung und Charakterisierung von Zerstäubung, Strahlausbreitung und Gemischbildung aktueller Dieseldirekteinspritzsysteme". PhD thesis, Universität Stuttgart.

[21] Gavaises, M., and Andriotis, A., 2006. "Cavitation Inside Multi-hole Injectors for Large Diesel Engines and Its Effect on the Near-nozzle Spray Structure". In 2006 SAE World Congress, SAE Technical Paper Series.

[22] Papoulias, D., Giannadakis, E., Mitroglou, N., Gavaises, M., and Theodorakakos, A., 2007. "Cavitation in Fuel Injection Systems for Spray-Guided Direct Injection Gasoline Engines". In 2007 World Congress, SAE Technical Paper Series.

[23] Oda, T., Iwatani, T., Takahashi, N., Sumi, T., and Ohsawa, K., eds., 2014. Behavior of Internal cavitating flow and primary spray break-up of a large scaled VCO Diesel nozzle with a small-lifted and eccentric needle.

[24] Soteriou, C., Andrews, R., Torres, N., Smith, M., and Kunkulagunta, R., 2001. "Through the diesel nozzle hole: A journey of discovery II". In ILASS - Europe 2001, European Conference on Liquid Atomization and Spray Systems, 2 -6 September 2001, Zürich, Switzerland, pp. 297-305.

[25] Kubitschek, J. P., and Weidman, P. D., 2007. "Helical instability of a rotating viscous liquid jet". Physics of Fluids, 19(11), p. 114108.

[26] Kirsch, V., Reddemann, M., Thalheim, B., Palmer, J., and Kneer, R., 2016. "Laser correlation velocity measurements in the near field of a diesel injector for variable ambient densities". In 4th TMFB International Conference, Aachen, Germany, 21-23 June 2016.

[27] Ghiji, M., Goldsworthy, L., Brandner, P. A., Garaniya, V., and Hield, P., 2016. "Numerical and experimental investigation of early stage diesel sprays". Fuel, 175, pp. 274-286.

[28] Lefebvre, A. H., 1989. Atomization and sprays. Taylor \& Francis, New York. 